Jährlich Grippeschutz ab 60 Jahren

\section{Regelmäßig Grippeimpfen erhöht die Schutzwirkung}

\author{
Mit dem Alter schwächt sich die Wirksamkeit der \\ Influenzaimpfung ab. Gerade deshalb ist es wichtig, \\ ältere Patienten Jahr für Jahr zu impfen.
}

90\% der influenzabedingten Todesfälle betreffen ältere Menschen. Weil ihre Immunabwehr nachlässt, sind sie anfälliger für Infektionen mit Influenzaviren und für schwere Krankheitsverläufe. Die Immunseneszenz ist auch der Grund dafür, dass die Grippeimpfung bei ihnen nicht mehr so gut anschlägt wie bei jüngeren Erwachsenen. Statt einer Schutzrate von $70-90 \%$ wird nur eine Quote von 30-40\% erreicht, wie Professor Janet McElhaney (Universität Sudbury) beim europäischen Pneumologenkongress berichtete. „Vor allem Influenza-A/H3N2-Viren sind für alte Menschen gefährlich“, so die Ärztin. Gegen A/H1N1Viren falle die Immunantwort robuster aus; vermutlich weil sie mit diesem Virusstamm schon in der Kindheit Bekanntschaft gemacht haben. „H1N1 ist die Krankheit der Jungen“, so McElhaney.

Die Prognose älterer Menschen ist allerdings weniger von den Lebensjahren als von den damit verbundenen Komorbiditäten abhängig. „Ein früherer Krankenhausaufenthalt wegen einer Pneumonie wird als Risikofaktor für Pneumonie oder Tod durch eine Influenza höher bewertet als ein Alter von 90 Jahren." Besser als das Alter allein ist laut McElhaney der Frailty Index geeignet, um die Prognose von älteren Influenzakranken abzuschätzen.

\section{Vorsicht: Im höheren Alter verläuft eine Influenza oft atypisch symptomarm}

Die hohe Komplikationsrate bei älteren Menschen hängt der Pneumologin zufolge auch damit zusammen, dass die Erkrankung unterdiagnostiziert ist. Im Alter seien häufiger atypische Verläufe zu beobachten: „Die Patienten haben öfter nur lokale Symptome und kein Fieber". Das führe dazu, dass die Gelegenheit einer frühen antiviralen Therapie versäumt werde. Durch den Beginn einer virostatischen Behandlung an Tag 1 bis 2 kann laut einer retrospektiven Studie das Risiko für ein Atemnotsyndrom und Tod gesenkt werden.

McElhaney ist eine „100\%ige Befürworterin“ der alljährlichen Impfung. „Es gibt sogar Daten, dass nach wiederholten Impfungen ein besserer Schutz besteht als nach der erstmaligen Impfung". Wenn es die epidemiologische Situation erlaubt, sei in Europa eine Impfung erst im Oktober/November möglicherweise günstiger als im September, weil dann auch im März noch genügend Antikörper produziert würden. Zweimal pro Saison den Impfstoff zu geben, ist der Expertin zufolge aber keine gute Idee. Vor allem die zellvermittelte Immunantwort werde dadurch möglicherweise sogar unterdrückt.

Dr. Beate Schumacher

Symposium "Influenza and respiratory physicians", Kongress der Eruopean Respiratory Society, London, 3.-7. September 2016

\section{Zusammenhang}

Ständig müde - zu viele Schadstoffe in der Luft?

Wenn sich im Büro regelmäßig Müdigkeit einstellt und die Augen zuzufallen drohen, mag das viele Gründe haben. Ein bisher kaum beachteter: die Belastung der Atemluft durch Autoabgase.

Nicht nur der Lärm, auch die Luftverschmutzung, die durch Straßenverkehr verursacht wird, kann ein Auslöser für Tagesschläfrigkeit sein. Darauf deutet eine epidemiologische Studie hin, die beim europäischen Pneumologenkongress in London vorgestellt wurde. Die Studienautoren um Ane Johannessen von der Universität Bergen raten, bei der Behandlung von Patienten mit Schlafstörungen auch den Einfluss des Straßenverkehrs zu berücksichtigen.

In der Studie RHINE (Respiratory Health in Northern Europe) III sind mehr als 12.000 Erwachsene zu Schlafstörungen und Verkehrsexposition befragt worden. Um das Ausmaß der Verkehrsexposition zu erfassen, sollten sie angeben, ob ihr Schlafzimmer nahe einer befahrenen Straße liegt, ob darin Straßenlärm zu hören ist und ob sie mehr als 60 Minuten pro Tag auf verkehrsreichen Straßen unterwegs sind. Die Fragen wurden von 10\%, $6 \%$ und $11 \%$ der Teilnehmer bejaht. Gleichzeitig berichteten $21 \%$ über Tagesschläfrigkeit. Das Risiko für Tagesschläfrigkeit war umso höher, je mehr jemand Autoabgasen ausgesetzt waren. Bei einer Exposition in der Nacht und am Tag lag es um 65\% höher als bei fehlender Exposition. Dabei war der Einfluss bekannter Risikofaktoren wie Rauchen, BMI, körperliche Aktivität, Schlafdauer und obstruktive Schlafapnoe berücksichtigt worden. Weniger überraschend war auch die nächtliche Lärmbelastung durch nahe gelegene Straßen mit dem vermehrten Auftreten von Tagesschläfrigkeit assoziiert. Die bereinigte Risikosteigerung betrug hier 46\%. Die Wahrscheinlichkeit, dass ein Studienteilnehmer Schnarcher war, korrelierte dagegen nur mit dem Verkehrslärm im Schlafzimmer (+29\%), nicht aber mit der Belastung durch Autoabgase.

Johannessen und ihre Kollegen raten, bei der Therapie von Schlafstörungen auch die Verkehrsexposition einzubeziehen. „Die Belastung zu reduzieren, indem man z. B. das Schlafzimmer verlegt, weiter weg von der Quelle der Luftverschmutzung, oder es besser gegen Geräusche isoliert oder indem man für die täglichen Routen im Freien weniger durch Abgase belastete Alternativen wählt, kann helfen."

Johannessen $A$ et al. Exposure to traffic pollution is related to daytime sleepiness and habitual snoring. ERS-Kongress, London, 3.-7. 9.2016

\section{Unfallgefahr}

\section{Schlafapnoiker kommen öfter vom Weg ab}

Wenn nächtliche Atemaussetzer den erholsamen Schlaf verhindern, dann ist es schwierig, tagsüber konzentriert und wach zu bleiben. Schlechte Basis also, um sich hinter das Steuer eines Autos zu setzen. Genau das zeigt auch eine Fahrsimulator-Studie, die beim ERS-Kongress vorgestellt wurde.

129 unbehandelte Patienten mit obstruktivem Schlafapnoe-Syndrom (OSAS) absolvierten jeweils 2 Fahrten und 79 gesunde Personen jeweils eine Fahrt im Simulator. Dabei wurde beurteilt, wie gut die Probanden die Spur hielten. Obwohl alle Teilnehmer im Mittel seit über 30 Jahren den Führerschein besaßen, hatten OSAS-Patienten mehr Probleme; die Standardabweichung von der Fahrspur war signifikant größer als in der Kontrollgruppe. Daher fielen 20\% beim Fahrtest durch, von den Kontrollen keiner. Die OSAS-Patienten hatten auch öfter berichtet, tagsüber einzunicken und beim Fahren schläfrig zu werden. Die Ergebnisse sind keine Überraschung, für Patienten mit OSAS wird in der Literatur ein 2- bis 6-fach erhöhtes Unfallrisiko im Straßenverkehr angegeben. Der Fahrsimulatortest könnte aber, so die Forscher, helfen, jene zu identifizieren, die tatsächlich hinterm Steuer zur Gefahr werden und sich daher einer CPAP-Therapie unterziehen sollten. bs

ERS-Kongress London, 3.-7.9.2016, Abstract \#2505 